\section{'HUATABAMPO ORO C2009', NUEVA VARIEDAD DE TRIGO CRISTALINO PARA EL NOROESTE DE MÉXICO}

\section{'HUATABAMPO ORO C2009', NEW DURUM WHEAT CULTIVAR FOR NORTHWEST MÉXICO}

\author{
Guillermo Fuentes-Dávila*, Pedro Figueroa-López, \\ Víctor Valenzuela-Herrera, Gabriela Chávez-Villalba, \\ J. Luis Félix-Fuentes y J. Alberto Mendoza-Lugo
}

Campo Experimental Norman E. Borlaug, Centro de Investigación Regional del Noroeste, Instituto Nacional de Investigaciones Forestales, Agrícolas y Pecuarias, Km 12 Norman E. Borlaug, Apdo. postal 155. 85000, Cd. Obregón, Sonora. Tel. 01 (644) 4145700 Ext. 254.

${ }^{*}$ Autor para correspondencia (fuentes.guillermo@inifap.gob.mx)

La producción mundial de trigo (Triticum sp.) en el año 2009 fue de 686.6 millones de toneladas (FAOSTAT, 2011a). China fue el mayor productor (115.1 millones), seguido por la India (80.6 millones); México produjo 4.1 millones de toneladas, de las cuales exportó 1.1 de trigo cristalino (FAOSTAT, 2011b). De la superficie total sembrada con trigo en el ciclo otoño-invierno 2008-2009 (457 419 ha) en el país, en los Estados de Sonora, Baja California, Sinaloa y Baja California Sur se sembró $64 \%$, con un valor de la producción de 4356 millones de pesos (SIAP, 2009). En esta región, el trigo es de hábito de crecimiento de primavera y se cultiva en condiciones de riego.

En México, el trigo duro o cristalino se produce principalmente en el Estado de Sonora, donde a partir del ciclo 2001-2002 ocupó poco más de 70 \% de la superficie sembrada (190 718 ha) (Camacho-Casas et al., 2004). La preferencia por esta clase de trigo se debe a su alto potencial de rendimiento, aceptación en el mercado de exportación, y su tolerancia al carbón parcial (Tilletia indica Mitra) y a la roya de la hoja (Puccinia triticina Eriksson). La variedad de trigo cristalino más sembrada en la región, hasta el ciclo 2002-2003, fue 'Altar C84', a pesar de que su resistencia a la roya de la hoja ya había sido vencida por la raza BBG-BN (Singh et al., 2004), la cual disminuyó su producción en los ciclos 2000-2001 y 2001-2002 (Figueroa-López et al., 2002; Com. Personal ${ }^{1}$ ).

La variedad 'Júpare C2001' (Camacho-Casas et al., 2004) dominó el área dedicada al trigo en el sur de Sonora desde

${ }^{1}$ Figueroa-López P, L A Gaxiola-Verdugo, A Suárez-Beltrán, R ÁlvarezZamorano, M A Camacho-Casas (2002) Análisis comparativo de las epidemias de roya de la hoja de trigo cristalino en el Valle del Yaqui, Sonora, México, en los años 2001 y 2002. Mem. XXIX/V Congreso Nacional/Internacional de la Soci. Mex. de Fitopatología. Julio 2 al 5 de 2002, Monterrey, Nuevo León, México. Resumen F-73. el 2003-2004 hasta el 2008-2009, donde se sembró en 119 327 ha (42.3\%), pero su resistencia también sucumbió ante otra nueva raza de roya (BBG-PN). En consecuencia, ha sido necesario diversificar las fuentes genéticas de resistencia para esta enfermedad así como incrementar el pigmento del grano, característica importante de calidad para el mercado de la exportación.

Como resultado de los trabajos de mejoramiento genético y de evaluaciones del rendimiento de grano llevadas a cabo a partir del ciclo otoño-invierno 2007-2008 en el Campo Experimental Norman E. Borlaug (CENEB), perteneciente al Instituto Nacional de Investigaciones Forestales, Agrícolas y Pecuarias, se propuso la liberación de la variedad de trigo cristalino 'Huatabampo Oro C2009' (GUAYACAN INIA/ POMA_2//SNITAN/4/D86135/ACO89//PORRON_4/3/ SNITAN), que presenta características agronómicas parecidas a las del testigo 'Júpare C2001', pero con mayor rendimiento de grano.

'Huatabampo Oro C2009' es una variedad de trigo duro o cristalino de hábito de crecimiento primaveral, la cual se obtuvo por hibridación y selección a través del método masal modificado, a partir de la cruza GUAYACAN INIA/

POMA_2//SNITAN/4/D86135/ACO89//PORRON_4/3/ SNITAN, llevada a cabo por el Centro Internacional de Mejoramiento de Maíz y Trigo (CIMMYT). Su número de cruza e historial de selección es CDSS02B00562S-0Y-0M2Y-1M-04Y-0B. Las selecciones individuales y en masa se efectuaron alternadamente en las estaciones experimentales de El Batán, Edo. de México (B) (19³0’ LN, 2249 msnm), San Antonio Atizapán, Edo. de México (M) (19² $17^{\prime} \mathrm{LN}, 2640 \mathrm{msnm}$ ) y en el Campo Experimental Norman E. Borlaug en el Valle del Yaqui, Sonora (Y) (27 $20^{\prime} \mathrm{LN}$, $40 \mathrm{msnm}$ ). Las condiciones de humedad fueron de secano (temporal) regular en las estaciones del Centro de México y riego normal en el Valle del Yaqui. 'Huatabampo Oro C2009' cuenta con el registro TRI-117-270510 del Catálogo Nacional de Variedades Vegetales (CNVV) del Servicio Nacional de Inspección y Certificación de Semillas (SNICS).

Las evaluaciones de rendimiento y calidad industrial de 'Huatabampo Oro C2009' se llevaron a cabo en los ciclos agrícolas 2007-2008 y 2008-2009 en el Campo Experimental Norman E. Borlaug. El rendimiento de grano que se obtuvo en varias fechas de siembra $\left(6.3 \mathrm{t} \mathrm{ha}^{-1}\right)$, fue $681 \mathrm{~kg}$ superior al testigo 'Júpare C2001'. Las mejores fechas para sembrar esta variedad son entre el 15 de noviembre y el 1 de diciembre, ya que su rendimiento disminuye significativamente en las siembras tardías del 15 de diciembre al 1 de enero. Esta variedad superó en rendimiento a 'Júpare C2001' en un poco más de $12 \%$ con dos o tres riegos de auxilio. En un lote de validación efectuado durante el ciclo agrícola 2009-2010 en el Valle del Mayo (SEMAY), 'Huatabampo Oro C2009' 
mostró un potencial de rendimiento promedio de $7.6 \mathrm{t} \mathrm{ha}^{-1}$ y un máximo de $8.8 \mathrm{tha}^{-1}$.

'Huatabampo Oro C2009' tiene una altura promedio de $86 \mathrm{~cm}$, ciclo de $76 \mathrm{~d}$ a espigamiento y de $118 \mathrm{~d}$ a madurez fisiológica (Figura 1). Presenta una fuerte coloración de antocianina en el coleóptilo y muy baja o nula frecuencia de plantas con hoja bandera curvada. La espiga tiene un perfil piramidal, con densidad media y longitud mediana (8.0 a $8.5 \mathrm{~cm}$ ); produce de 19 a 21 espiguillas, y cada espiguilla produce de tres a cinco granos en el tercio inferior y tercio medio de la espiga, y de una a cuatro en el tercio superior. En el tercio medio de la espiga, el hombro de la gluma es angosto y de forma redondeada, con una punta corta de forma ligeramente curva y con vellosidad en la superficie externa. Su grano es de color ámbar, elíptico, de $7.6 \mathrm{~mm}$ de longitud y $3.2 \mathrm{~mm}$ de ancho (Figura 2), con un peso medio de $69.0 \mathrm{mg}$ un peso específico medio de $83.6 \mathrm{~kg} \mathrm{hL}^{-1}$; su cantidad varía entre 10036 y 11008 granos $/ \mathrm{m}^{2}$. El color del grano puede ser muy tenue o estar ausente al aplicarle fenol.

Mientras que 'Júpare C2001' es susceptible a la raza BBGPN de roya de la hoja, 'Huatabampo Oro C2009' es resistente. Ambas variedades son resistentes a la roya amarilla o lineal, pero 'Huatabampo Oro C2009' es moderadamente resistente al carbón parcial mientras que 'Júpare C2001' es resistente. 'Huatabampo Oro C2009' presenta un promedio de $13.9 \%$ de proteína en grano y 27.5 puntos en la escala b de Minolta.

El Campo Experimental Norman E. Borlaug mantiene las categorías de semilla original y básica de esta variedad. La semilla registrada se ha incrementado a través del Patronato para la Investigación y Experimentación Agrícola en el Estado de Sonora (PIEAES). La siembra de 'Huatabampo Oro C2009' se recomienda para el ciclo de producción otoñoinvierno en la región del noroeste de México.

\section{AGRADECIMIENTOS}

A la Coordinadora Nacional de Fundaciones Produce, A. C. (proyecto 40-2007-0900) y al Patronato para la Investigación y Experimentación Agrícola en el Estado de Sonora (PIEAES), por el financiamiento parcial de los trabajos de investigación que condujeron a la obtención de la variedad 'Huatabampo Oro C2009'. También al Centro Internacional de Mejoramiento de Maíz y Trigo (CIMMYT), por proporcionar las líneas avanzadas de donde se originó la variedad.

\section{BIBLIOGRAFÍA}

Camacho-Casas M A, P Figueroa-López, J Huerta-Espino (2004) Júpare C2001, nueva variedad de trigo duro para su cultivo en el noroeste de México. Folleto Técnico No. 47. INIFAP-CIRNO, Campo Experimental Valle del Yaqui. Cd. Obregón, Sonora, México. 16 p.

FAOSTAT (2011a) World Wheat Production 2009. Disponible en: http://faostat.fao.org/site/567/DesktopDefault.aspx?PageID=567\#ancor. (Diciembre 2011).

FAOSTAT (2011b) Imports, Exports, Commodity by country 2009. Disponible en: http://faostat. fao.org/site/342/default.aspx. (Diciembre 2011)

SIAP, Servicio de Información Agroalimentaria y Pesquera (2009) Secretaría de Agricultura, Ganadería, Desarrollo Rural, Pesca y Alimentación (SAGARPA). Anuario Estadístico de la Producción Agrícola. Disponible en: http://www.siap.gob.mx. (Mayo 2011).

Singh R P, J Huerta-Espino, W Pfeiffer, L P Figueroa (2004) Occurrence and impact of a new leaf rust race on durum wheat in the Northwestern Mexico during 2001-2002. Plant Dis. 88:703708 . 


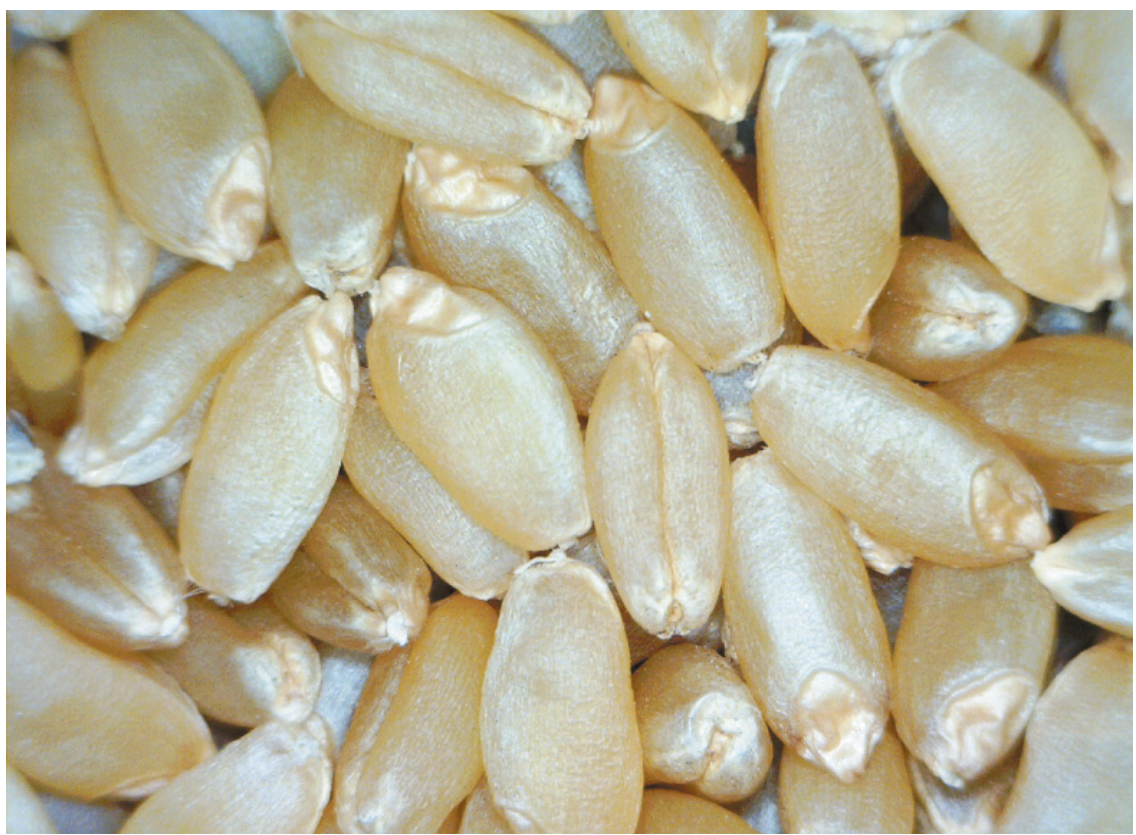

Figura 2. Grano cristalino de la variedad 'Huatabampo Oro C2009' .

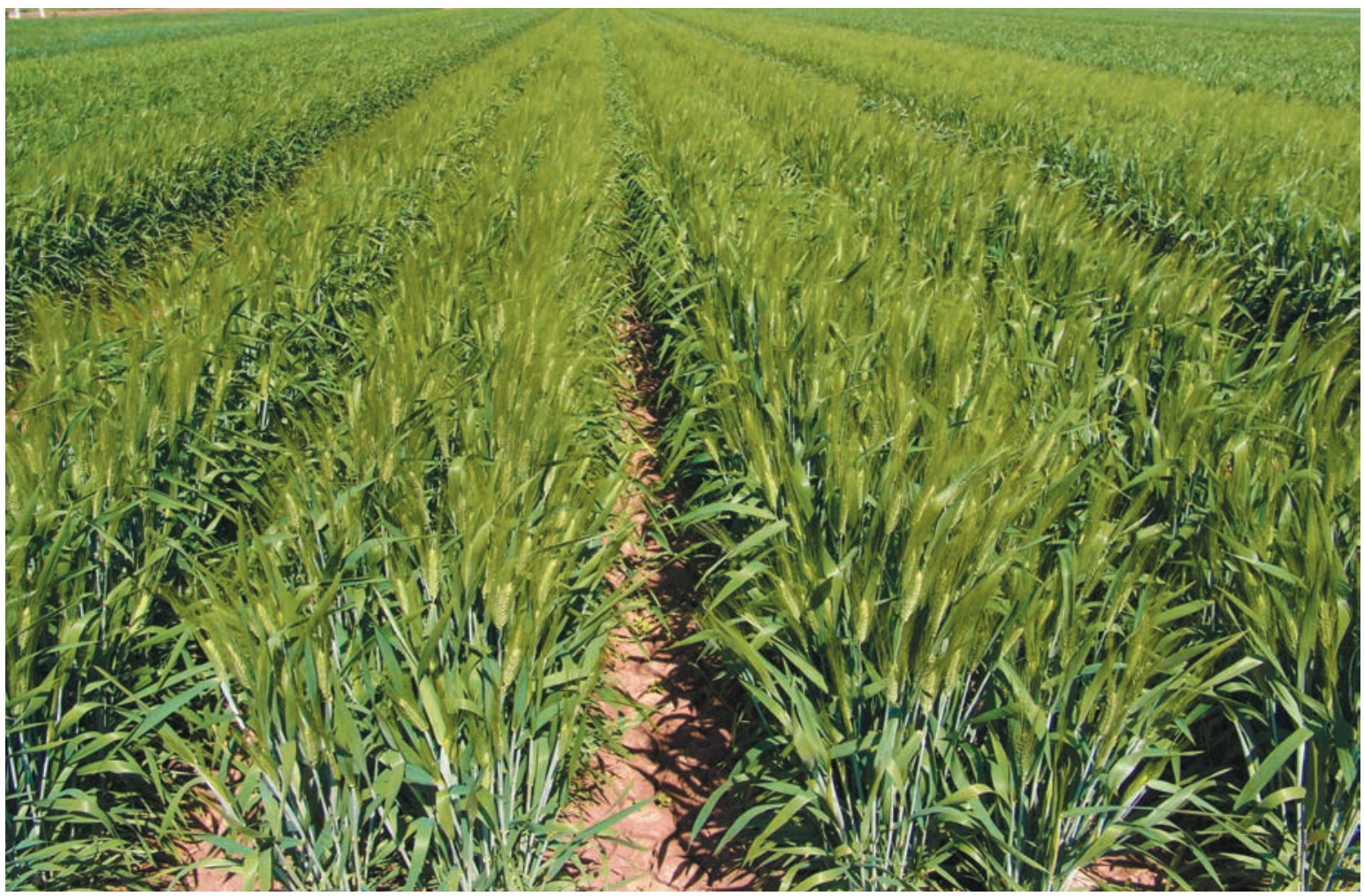

Figura 1. Planta de la variedad de trigo 'Huatabampo Oro C2009'. 